Spring 4-25-2020

\title{
Non-pharmaceutical Methods for Pain Management: An Occupational Therapist's Perspective on Chronic Pain
}

\author{
Alison L. Brown \\ University of St. Augustine for Health Sciences \\ DOI: https://doi.org/10.46409/sr.BGXI5404
}

Follow this and additional works at: https://soar.usa.edu/capstones

Part of the Occupational Therapy Commons, and the Other Rehabilitation and Therapy Commons

\section{Recommended Citation}

Brown, A. L. (2020). Non-pharmaceutical Methods for Pain Management: An Occupational Therapist's Perspective on Chronic Pain. [Doctoral project, University of St Augustine for Health Sciences]. SOAR @ USA: Student Capstone Projects Collection. https://doi.org/10.46409/sr.BGXI5404

This Capstone is brought to you for free and open access by the Student Research at SOAR @ USA. It has been accepted for inclusion in Student Capstone Projects by an authorized administrator of SOAR @ USA. For more information, please contact soar@usa.edu, erobinson@usa.edu. 
Non-pharmaceutical Methods for Pain Management: An Occupational Therapist's Perspective on Chronic Pain

By Alison L Brown

A Capstone Presented in Partial Fulfillment

of the Requirement for the Degree of DOCTOR OF OCCUPATIONAL THERAPY

University of St. Augustine for Health Sciences

April, 2020 
Non-pharmaceutical Methods for Pain Management: An Occupational Therapist's Perspective on Chronic Pain

by

Alison L Brown

has been approved

April, 2020

APPROVED:

Kayla Collins, EdD, OTR/L, Doctoral Coordinator

Thais Petrocelli, OTD, OTR/L, Doctoral Coordinator

Mary Shotwell, PhD, OT/L, Program Director

ACCEPTED AND SIGNED:

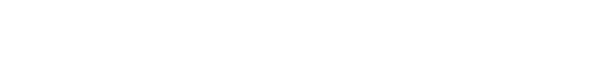

Kayla Collins, EdD, OTR/L, Doctoral Coordinator

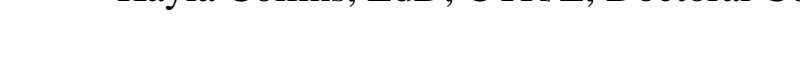

Thais Petrocelli, OTD, OTR/L, Doctoral Coordinator

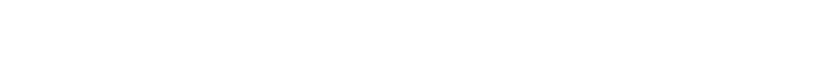

Mary Shotwell, PhD, OT/L, Program Director 


\section{Table of Contents}

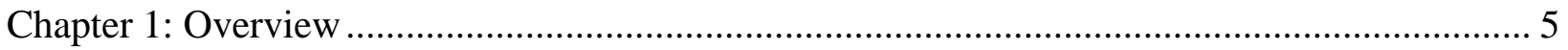

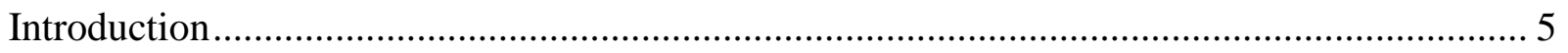

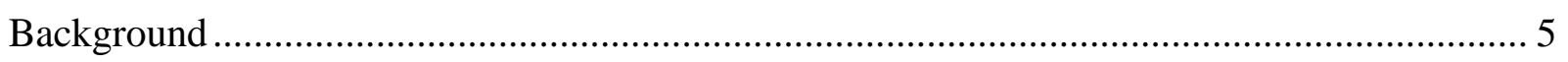

Statement of Problem............................................................................................... 7

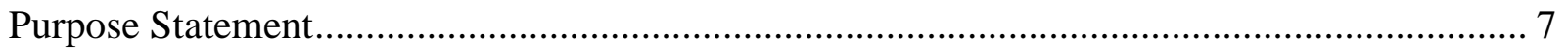

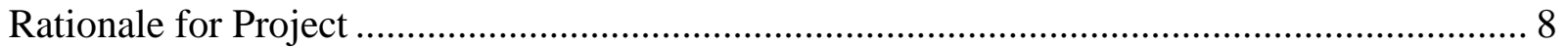

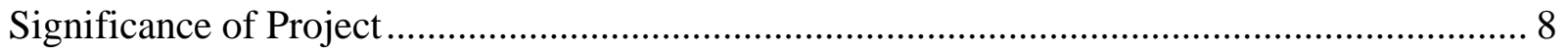

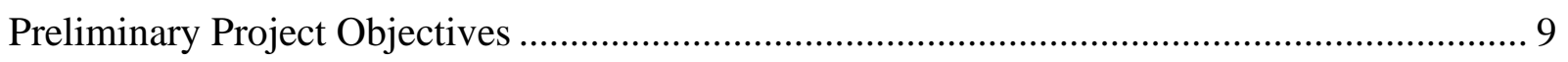

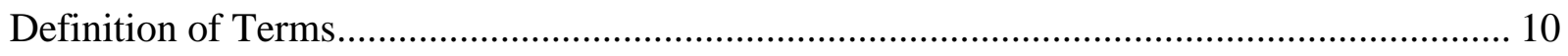

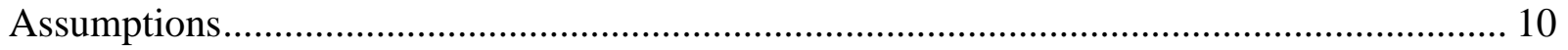

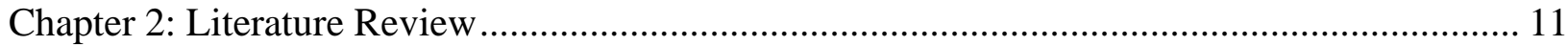

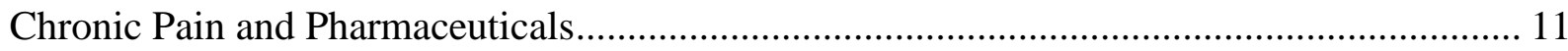

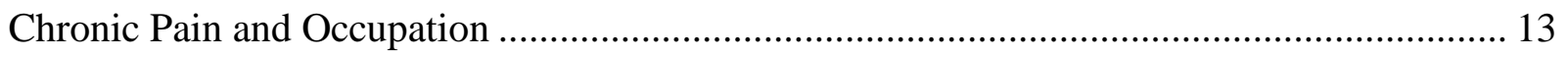

A Nonpharmaceutical Approach............................................................................ 15

Occupational Therapy Interventions ........................................................................ 16

Ergonomics, Body Mechanics, and Posturing ............................................................ 16

Adaptive Equipment and Adaptive Strategies ......................................................... 17

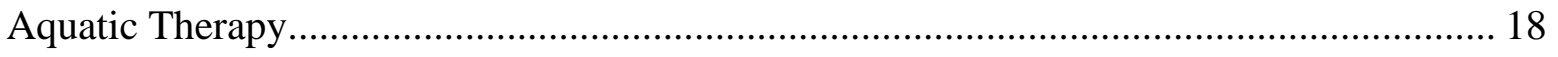

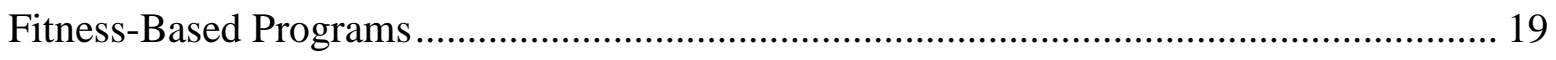

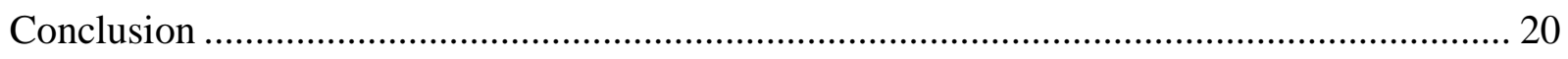

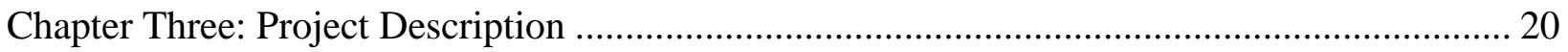

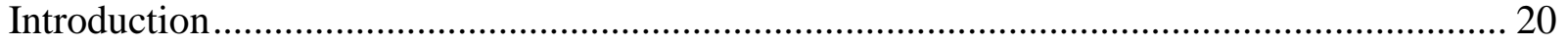

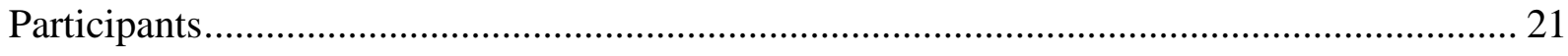

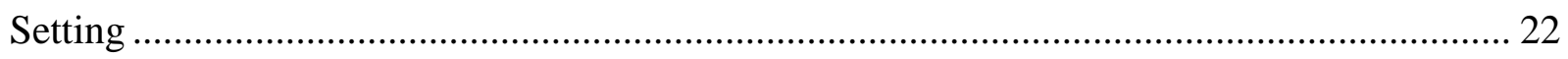

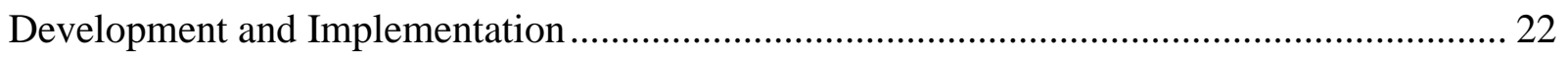

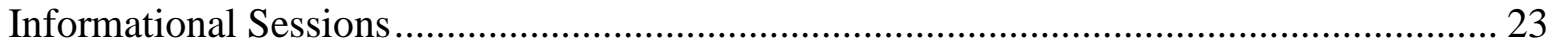

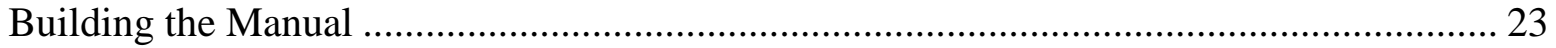

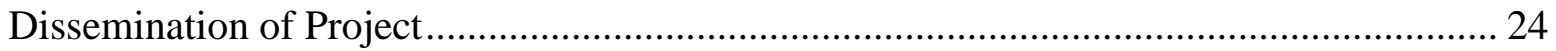

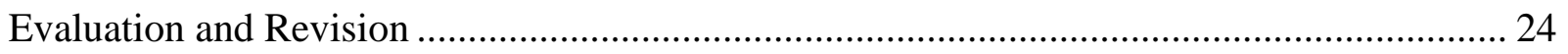

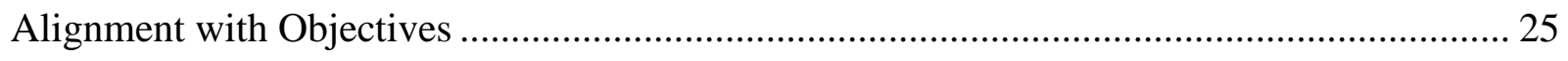

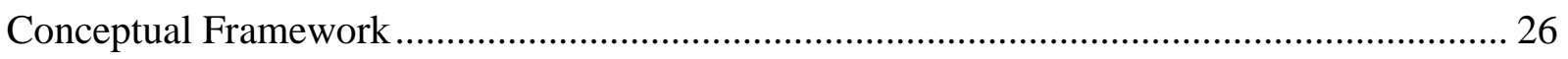




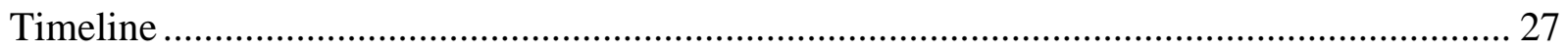

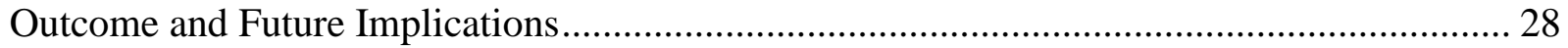

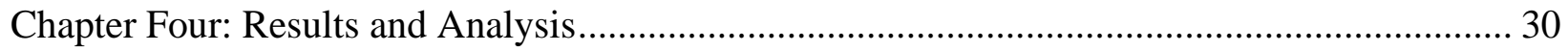

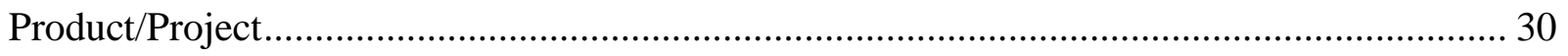

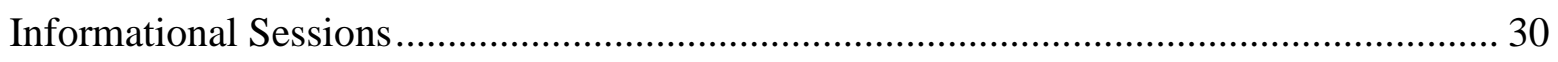

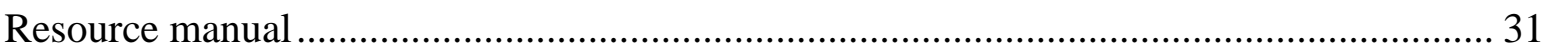

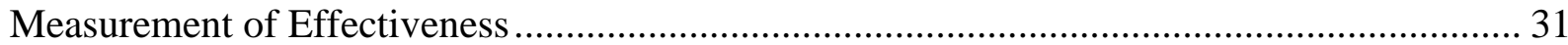

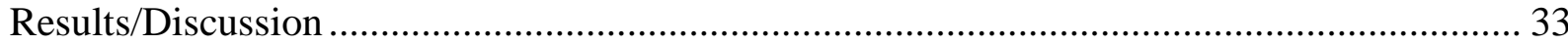

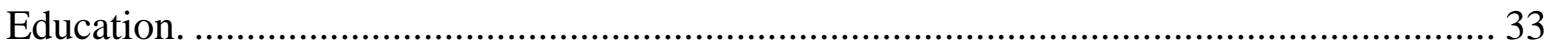

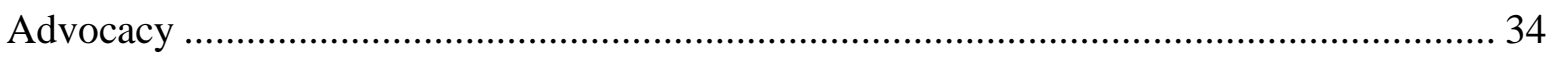

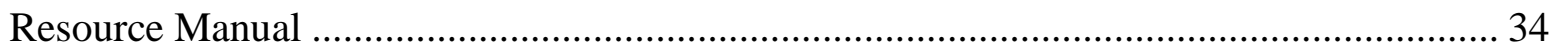

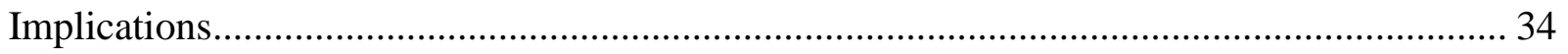

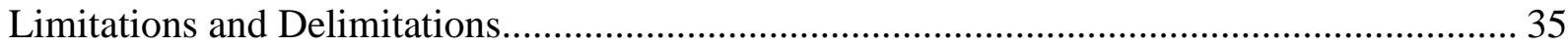

Chapter Five: Summary, Conclusions, and Discussion ...................................................... 35

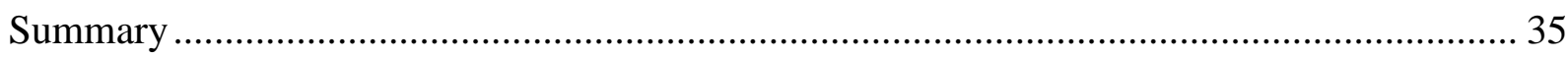

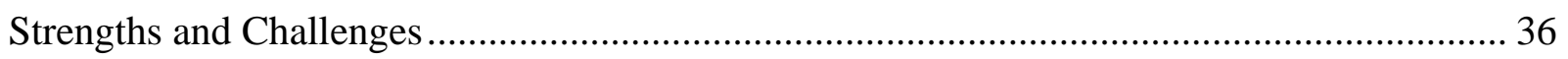

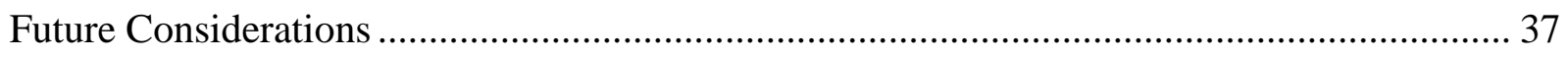

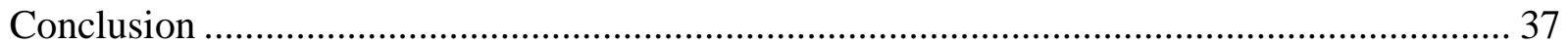

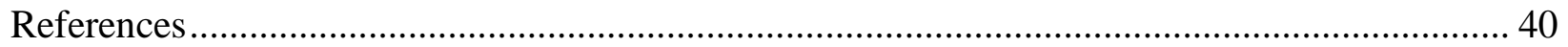




\section{Chapter 1: Overview}

\section{Introduction}

Chronic pain in the United States (U.S.) is one of the most common reasons to seek medical advice (Simsek, Evli, \& Uzdil, 2019). It has been associated with depression, anxiety, poor perceived health, lowered quality of life (QOL), and opioid dependence (Dahlhamer, et al., 2018). In 2017, the U.S. alone, approximately 50 million people were diagnosed with chronic pain, with an additional 20 million diagnosed with high-impact chronic pain. During that year, about 58.5 out of every 100 prescriptions were written for opioids. These numbers were denser in counties found to have higher rates of chronic illnesses (Center for Disease Control and Prevention [CDC], 2019). That means in the year 2017, approximately 40.9 million Americans were written an opioid prescription.

Despite efforts for chronic pain to be relieved by various pharmaceuticals, many patients with chronic pain report medications only help temporarily or can only provide slight relief to their pain levels (Hadlandsmyth, et al, 2018). This capstone project focused on occupational therapy's role in the management of chronic pain through a non-pharmacological approach. The expected outcome of this project was to provide a practical evidence-based resource to patients and clinicians that explained occupational therapy's role in chronic pain. Informational sessions were also provided that discussed the research based non-pharmaceutical interventions to various audiences to advocate for occupational therapy's role in chronic pain.

\section{Background}

Occupational therapy (OT) is a practice that wears many hats. Therapists can work with a vast array of facilities, diseases, and populations. An occupational therapist's main goal is to provide a way for individuals to maintain their quality of life through engagement in functional 
everyday activities. Occupational therapists specialize in helping people gain their independence back after an injury, during present developmental delays, or throughout the course of chronic diseases. Chronic pain is often coupled with having a chronic disease. The management of chronic pain is another facet for occupational therapist's to work in.

Chronic pain is defined by pain persisting longer than 3 months and can be a side effect from an injury, a disease/illness, medical malpractice, or other accident/trauma (Hesselstrand, Samuelsson, \& Liedberg, 2015). People living with chronic pain issues can also have feelings of suffering and social isolation, trouble maintaining employment, difficulties with activities of daily living (ADLs), and lower QOL. Chronic pain can be comorbid with other diseases or ailments causing multiple levels of pain and is also a risk factor for premature death (Domenichiello \& Ramsden, 2019). Upon initial evaluation, the occupational therapist must look at the whole patient and make note of any underlying diseases, past injuries, or difficulties the patient has been having.

When patients are experiencing chronic pain, it raises the need for pain relief. The most common practice for this is the use of pharmaceuticals. Currently, America is in the "opioid crisis." The opioid crisis is characterized by misuse, overdose, or death caused by prescription opiates or synthetic opioids. Examples of opioids include codeine, morphine, fentanyl, heroin, and many others. Opioids are not the only problem; however, opioids are only one type of drug others include antibiotics, diuretics, anticoagulants, hypoglycemics, steroids, benzodiazepines, and nonsteroidal anti-inflammatories just to name a few that can also have side effects on a person (CDC, 2019). However, these drugs cannot fix or heal the problem but only temporarily soothe the pain, thus only being partially effective (Domenichiello \& Ramsden, 2019). That is where occupational therapy comes into the picture. Non-pharmacological approaches such as 
using transcutaneous electrical nerve stimulation (TENS), hot or cold compresses, relaxation techniques, massage, and music therapy are just a few examples of techniques to try with patients experiencing chronic pain (Yaban, 2019). Occupational therapists specialize in creating the most conducive environment for a patient to live successfully. The same is true of incorporating pain management techniques to aid in allowing patients to be more independent and increase their QOL.

\section{Statement of Problem}

The utilization of pharmaceuticals does not rid the patient of pain completely; it typically just mutes the pain or dulls it for a few hours, and they often come with other side-effects, too (Domenichiello \& Ramsden, 2019). Some of the side-effects include sedation, constipation, cognitive dysfunction, nausea/vomiting, lack of sexual desire, and dizziness (Birke, Ekholm, Hojsted, Sjogren, \& Kurita, 2018). By not directly addressing the pain itself and finding more effective ways to manage it, our patients are still feeling the pain and the effects of the medication on their body without any real relief. Occupational therapists are prepared to address the needs of chronic pain. However, there is no relevant literature to educate clinicians on the use of non-pharmacological pain relief techniques, or how to adequately incorporate them into everyday treatment sessions. By utilizing a non-pharmacological approach, occupational therapists can address factors like life satisfaction, physical and emotional problems, general health, energy and fatigue, and occupational performance (Lagueux, Depelteau, \& Masse, 2018).

\section{Purpose Statement}

The purpose of this capstone was to develop an evidence-based manual for patients and clinicians that outlined non-pharmacological approaches to manage chronic pain symptoms. The manual focused on alternative methods that had evidence supporting their benefit in an array of 
chronic pain diagnoses. This project also provided informational sessions on nonpharmacological OT approaches to therapy staff, nursing staff, and residents of a skilled nursing facility (SNF) in Melbourne, Florida. An additional session was provided to the members of the Student Occupational Therapy Association (SOTA) at the University of Saint Augustine. These sessions provided the clients and clinicians with information about occupational therapy's role in chronic pain and research-based examples of alternative options for pain. The research demonstrated the efficacy of non-pharmacological approaches in chronic pain. This capstone aimed to put them into a useful resource for clinicians and patients to advocate for, and better understand, occupational therapy's role in chronic pain.

\section{Rationale for Project}

Occupational therapists are trained to evaluate how pain influences the way a patient interacts with their environment and how it affects their daily occupations (Lagueux, et al., 2018). The problem with chronic pain is that it limits the patient's ability to complete everyday tasks and can affect sleep, appetite, and quality of life. Chronic pain influences many occupations. Through OT, therapists can use non-pharmacological approaches to adapt a task, change the task demands, and alleviate pain so that their daily occupations will once again be possible (Hesselstrand, et al., 2015; Lagueux, et al., 2018). Being able to get patients at a more tolerable level of pain or to eliminate it allows patients to be more independent and increases their quality of life. Managing chronic pain needs to be something that becomes a norm for occupational therapists to assist in.

\section{Significance of Project}

Patients all over the country are living with lower quality of life, daily pains, and little to no relief. These patients have the potential to reduce their pain by incorporating simple 
techniques into their everyday lives using occupational therapy. However, due to under-informed clinicians and lack of research, occupational therapists have not been doing all they can to help eliminate patients' chronic pain symptoms. The idea is to inform other clinicians of how occupational therapy's role in chronic pain management can benefit their patients. While also providing examples of research-based interventions that occupational therapy can offer to their clients who have chronic pain.

In response to this information, the goal is to advocate for occupational therapy's role so that instead of just continuously trialing opioids or other drugs perhaps they will consider obtaining a therapy prescription for their patients instead. This means that the advocacy used for OT and chronic pain can assist in relieving pain and potentially lower the necessity for using prescription drugs. With the addition of a resource that would assist in the implementation of these chronic pain interventions, occupational therapists could help alleviate pain, increase overall QOL, decrease the need or frequency for pharmacological intervention, and help patients live more independent lives.

\section{Preliminary Project Objectives}

Outcome Objectives:

- Develop and disseminate educational resources for practicing clinicians on nonpharmaceutical pain management techniques.

- Provide at least three informational sessions to varying audiences demonstrating the benefits of OT on chronic pain, focused on educating participants on non-pharmaceutical pain management techniques.

- Conduct a needs assessment on chronic pain experienced by residents living at Melbourne Terrace Rehab 
- Create an evidence-based resource manual documenting the role of OT in reducing chronic pain through non-pharmacological pain management techniques.

- Review and revise the material presented in the client informational sessions based on participant feedback.

\section{Definition of Terms}

Chronic pain. Chronic pain is a pain that persists beyond 3 months or past the normal time of healing (Domenichiello \& Ramsden, 2019). In this capstone, the focus of this intervention was to minimize chronic pain symptoms utilizing non-pharmacological interventions.

Occupations. Occupations are any purposeful activities including self-care, productivity, and leisure (Hill, 2016). For this capstone, interventions aided clients in being more independent with their occupations.

Non-pharmacological interventions. Non-pharmacological interventions are methods of treatment other than drugs utilized to reduce pain (Yaban, 2019). These types of interventions were provided as education to the population to explain the benefits occupational therapy can have on chronic pain.

Opioids. Opioids are prescription drugs used to reduce pain (Opioid Overdose, 2019). In this capstone, the idea was to demonstrate an alternative to the sole use of prescription drugs for pain relief and to provide alternative suggestions with an evidence-based manual.

\section{Assumptions}

Assumptions of this capstone project were that participants in the informational sessions were willing participants and were seeking resources to support non-pharmaceutical chronic pain management. Another assumption is that the manual was an effective and useful resource for 
patients, therapy staff, nursing staff, and students to manage chronic pain, as well as was a relevant resource to those utilizing it.

\section{Chapter 2: Literature Review}

\section{Chronic Pain and Pharmaceuticals}

Chronic pain is recognized as a public health problem by the World Health Organization (WHO) and, more than just being recognized as a symptom, is also classified as a disease in the International Classification of Diseases (Lagueux, et al., 2018). One of the top reasons that patients seek medical attention is for chronic pain (Simsek, et al., 2019). Chronic pain is described as a pain that lasts longer than three months and affects an individual's ability to maintain an independent lifestyle (Gupta, Mohanty, \& Pattnaik, 2019). It is estimated that onethird of the people dealing with chronic pain are unable to maintain their independence and that two-thirds cannot complete their basic ADLs (Hesselstrand, et al., 2015). Chronic pain can involve the joints, muscles, bones, or nerves associated with different areas of the body. Chronic pain can be the result of a chronic condition, illness, disease, injury, or complication of surgery. A few examples of chronic pain are cancer, back problems, arthritis, nerve damage, or symptoms related to stroke (Vendrusculo-Fangel, Leles, Moura, Marqueti, \& Fangel 2019; Hadlandsmyth, et al., 2018). Pain is not subjective and does not discriminate; however, age is one of the most critical components associated with chronic pain (Stensland \& Saunders, 2018).

One of the most common initial treatment options for chronic pain is the use of pharmaceuticals. While there is some evidence suggesting that drugs can be a useful tool for the initial healing process, there is even more speaking against the use of pharmaceuticals as the only effective treatment method (Lagueux, et al., 2018; Mehta, et al., 2018). Pharmaceutical use in older adults typically only provides partial relief and is limited by side effects. Not to mention 
that the risk of side effects in seniors is exponentially higher due to polypharmacy drug interactions (Domenichiello \& Ramsden, 2019). Long-term use of opioids alone can cause sleep disorders; sexual dissatisfaction; excessive sedation; appetite disturbance; respiratory, bowel, and endocrine dysfunctions; opioid misuse and abuse; transitions to harder illicit drug use; and possible overdose (Hadlandsymth, et al., 2018; Mehta, Kaur, Smith, Mani, \& Baxter, 2018; Birke, et al., 2019).

A recurring theme associated with the use of pharmaceuticals brings up issues with the opioid crisis, overdosing, crippling side-effects, and overall loss of independence. The problem with treating patients solely with pharmaceutical methods is that it is only partially effective, and the actual source of pain typically is not addressed (Domenichiello \& Ramsden, 2019). Thus, relief is not being provided to its fullest potential. When measuring pain, it is highly subjective and individualized (Lagueux, et al., 2018). Taking a drug for pain relief does not correct any of the patient's limitations, musculoskeletal deficits, assess the need for adaptive equipment, or to increase patient independence (Dahl-Popolizio, Manson, Muir, \& Rogers, 2016). Thus, research demonstrates that chronic pain relief has a clear need to be more than just temporary.

Occupational therapy's role in chronic pain management is lacking due to not having enough literature supporting the efficacy of non-pharmaceutical interventions $(\mathrm{Wu}$, Meleger, Witkower, Mondale, \& Borg-Stein, 2015). Solutions that are longer-term and more effective need to be given as a tool to clinicians so that their patients can have increased independence and QOL. It can be assumed that clinicians and patients alike need to be educated in the benefits of choosing OT in place of, or in conjunction with, pharmaceuticals. Occupational therapy can help to close the gap using non-pharmaceutical pain management techniques (Lucio, Laney, Lake, Kornblau, \& Mbiza, 2018). 
Common themes throughout research of how pain affects older adults include their decreased independence, the stigma of being perceived as old, and dealing with the impact chronic pain has on their future (Stensland \& Sanders, 2018). Older adults who acquire an unforeseen condition that leaves behind chronic pain can completely change how they initially envisioned their current work situation, retirement, leisure activities and more. Chronic pain leads to a loss of social roles, inability to fulfill work roles, unmet expectations of the future, and dreams that feel unachievable due to limited mobility (Vendrusculo-Fangel, et al., 2019; Domenichiello \& Ramsden, 2019; Dahlhamer, et al., 2018). Another big concern from older adults living with chronic pain is the appearance of being old or helpless, or appearing like they have a deficit by using back braces, canes, or other assistive devices (Stensland \& Sanders, 2018). Occupational therapy can help to create a more effective method to approach chronic pain.

\section{Chronic Pain and Occupation}

Chronic pain is not just affecting the body negatively, but is also negatively affecting a person's daily activities, or occupations. Chronic pain and occupation are linked in more ways than one might consider at first glance. Chronic pain influences biological, psychological, and social factors and can affect individuals and their families (Amris, et al., 2019). Occupational therapists specialize in client-centered practices. Occupational therapists use the patient's interests, roles, values, and beliefs to create an environment that is conducive for maximizing independence (Hesselstrand, et al., 2015; Lagueux, et al., 2018). They are trained to use an individual's motivating factors to adapt, create, and modify tasks using task analysis to allow their patients to succeed, despite limitations they may be facing (Lagueux, et al., 2018). 
Studies have demonstrated the effectiveness of utilizing non-pharmacological approaches for chronic pain. One study by Simon \& Collins (2017) provided the clinical outcome of 45 patients after completing what they deemed "Lifestyle Redesign." The patients in the study developed healthy self-care routines and habits to manage chronic pain. Changes included making one's lifestyle functional, occupation-based, applicable to each patient, and reliable for follow through. Some examples are physical activity, energy conservation, body mechanics, and eating routines. This study added to the literature on lifestyle occupation-based intervention and provides support for improving the function of the individual (Simon \& Collins, 2017).

In addition, a scoping literature review by Lagueux et al. (2018) supports that OT’s unique approach to interventions enables a patient's independence by utilizing occupations that are interesting to the patient. The occupational therapists direct the focus of therapy and create an optimal environment for participating in everyday life roles (Lagueux, et al., 2018). Occupational therapists not only look at eliminating the pain but also increasing the person's functional ability to participate in everyday life. Simple techniques like task modifications, adaptive equipment, use of proper body mechanics, and exercises could change a person's ability to complete a task independently (Hesselstrand, et al., 2015; Hill \& Macartney, 2019). This may suggest that occupational therapists have a bigger role to play in chronic pain management than what is being utilized in current practice.

Lucio et al. (2018) presented data from a qualitative study of OT and familiarity with chronic pain treatments. The study concluded that practitioners were not adequately trained to handle these types of cases from just a conventional occupational therapy program. It mentions that there is a trend in finding non-pharmacological treatment interventions and that occupational therapists can make a bigger impact (Lucio, et al., 2018). Using an alternative 
nonpharmacological approach for pain management could allow for patients to decrease their pain instead of just masking its symptoms.

\section{A Nonpharmaceutical Approach}

Though there is a gap in treating chronic pain between school and everyday practice, occupational therapists have a responsibility to not only be informed but to inform others on OT's role in managing chronic pain (Lucio, et al., 2018). Currently there are therapists all over the country utilizing nonpharmaceutical techniques to adapt their patient's ADLs allowing for increased independence in their daily occupations (Hill, 2016). However, in the literature occupational therapy's role is rarely included in interdisciplinary pain programs and the effect pain has on daily activities is missing (Simon \& Collins, 2017). It could be assumed that there is a need for education on how vital occupational therapy's role can be in managing chronic pain with a non-pharmacological approach.

Occupational therapists have a responsibility to help the patients who despite their use of pharmaceuticals, are still experiencing severe pain that significantly impact their function (Hesselstrand, et al., 2015). There are patients who lose sleep because their pain won't allow them to rest, or those who won't eat because their pain is too high (Mehta, et al., 2018). Advocacy for occupational therapy can then provide patients with more options for relief. This includes interventions like massage, nerve/tendon glides, coping strategies, mindfulness, fitness programs, posturing/body mechanics, electrical stimulation, stress loading, breathing, and more (Lagueux, et al., 2018). Chronic pain appears to be such a daunting task, but by breaking down activities that cause the patients pain, occupational therapists can use task analysis to create a treatment plan to effectively reduce pain, increase independence, and quality of life (Lagueux, et al., 2018). This may be intimidating for clinicians to feel comfortable doing, which is why 
creating an evidence-based manual will allow for easy access to education on nonpharmaceutical solutions.

\section{Occupational Therapy Interventions}

Non-pharmaceutical interventions have proven their efficacy in recent literature. Nonpharmaceutical interventions are provided methods of treatment, other than drugs, utilized to reduce chronic pain in patients (Yaban, 2019). This project will focus on how occupational therapists can utilize body mechanics, adaptive equipment, adaptive strategies, aquatic therapy, and fitness programs to reduce chronic pain symptoms, increase independence, and quality of life (Atlay, Akova, Giir, \& Sekir, 2017; Gupta, et al., 2019; Hill, 2016; Simon \& Collins, 2017; Keane, 2016; Rochman, 2014; Wu, et al., 2015). These techniques form the foundation of the manual created in this capstone project that provides a practical tool for clinicians to utilize when treating chronic pain.

Ergonomics, Body Mechanics, and Posturing. Ergonomics is defined as the performance of a task within an environment (Hill, 2016). Body mechanics are the correctly aligned postures and movements necessary to complete the task safely (Hill, 2016). Teaching a patient on the use of safe posturing during the completion of a task can provide them with the opportunity for increased self-efficacy in their pain management. This is because they will learn firsthand the benefits posturing and proper body mechanics has on the reduction of bodily strain during occupational performance (Rochman, 2014). Body positioning is important during bending, lifting, transfers, and interaction in patient's daily environments (Simon \& Collins, 2017). It can be assumed that many people use a combination of these movements during everyday occupations. Ergonomic assessments can be utilized by the occupational therapist to assess a patient's form during a task to decide if there is an issue in the body mechanics of the 
patient (Rochman, 2014). While poor body mechanics may not be the cause of the chronic pain, correcting a patient's form can help to minimize the pain's impact during occupation (Hill, 2016).

Adaptive Equipment and Adaptive Strategies. The use of adaptive equipment and/or adaptive strategies for clients means that tasks can be simplified using tools, tricks, or tips to increase independence during occupations (Radomski \& Trombly Latham, 2014). Adaptive strategies include goal setting, energy conservation, pacing, and flare-up planning (Hill, 2016; Radomski \& Trombly Latham, 2014; Rochman, 2014; Simon \& Collins, 2017). Adaptive equipment can include assistive devices like button hooks, zipper pulls, long handled shoehorns, built up handles, bed side commodes, universal cuffs, shower chairs, and reachers (Radomski \& Trombly Latham, 2014). These strategies can all be useful in alleviating pain symptom in patients (Hill, 2016; Radomski \& Trombly Latham, 2014; Simon and Collins, 2017).

According to Hill (2016), goal setting can be utilized by patients who struggle with the realistic expectations of their disability caused by pain. Goal setting allows the patient to set goals that are more attainable given their limitations and provides the patient with increased selfefficacy when those goals are then met. Energy conservation addresses the fatigue of chronic pain and can also help a client to prioritize more important tasks or adapt them to make them less impactful on their fatigue. Pacing can also be used to break up activities into smaller chunks, so they are more easily managed by the individual (Hill, 2016).

Simon and Collins (2017) suggest the use of flare-up planning as a useful tool for individuals living with chronic pain. Flare-up planning identifies when flare-ups are most common, how often they happen, and what triggers them to occur. Being able to identify these factors will help to develop a "wellness toolbox" to maintain the symptoms appropriately or rest 
before they get worse. Finally, the use of assistive devices can help to adapt a task for the patient to help alleviate pain and complete the task more independently (Simon \& Collins, 2017).

Aquatic Therapy. Another topic for pain relief in recent literature is aquatic therapy. Aquatic therapy has literature that provides support to its' aid in chronic pain management. Studies have noted it being useful to relieve chronic pain symptoms for fibromyalgia, knee pain, and low back pain (Keane, 2016; McIlroy, Sayliss, Browning, \& Bearne, 2017; Zamuner, Andrade, Arca, \& Avila, 2019). In addition to relieving chronic pain symptoms, aquatic therapy has also been noted to be effective in increasing quality of sleep while also reducing stress and pain sensitivity (Zamuner, et al., 2019). Aquatic therapy combines water's buoyancy, resistance, and heat to assist in exercise performance (McIlroy, et al., 2017). Aquatic therapy provides an environment where gravity's weight on the body is reduced and can in turn improve range of motion, strength, and movements (Zamuner, et al., 2019). It can also cause muscles to relax which can help in the reduction of pain throughout the body (Zamuner, et al., 2019).

In a study by Keane (2016), an aquatic therapy program called AquaStretch was compared to land-based stretching for reduction of chronic lower back pain. Land-based stretching is a series of whole body stretching completed on a flat surface. The AquaStretch program is a set a stretching completed in a therapy pool. The study was completed 30 minutes twice a week for twelve weeks. Though both groups demonstrated effectiveness, the land-based stretching group's progress plateaued after 6 weeks, while the group in the pool continued to show signs of improvement. The goal of this study was to monitor the reduction rates of pain, disability, and kinesiophobia (fear of pain from movement); this study was successful at reducing these factors (Keane, 2016). It can be assumed that the benefits of aquatic therapy can influence the whole body in relation to pain relief. 
Fitness-Based Programs. Finally, fitness-based programs have also demonstrated efficacy in recent literature. Fitness-based programs are any activities that relate to exercise. According to Gupta, et al., (2019), people living with chronic pain often begin to avoid exercise secondary to fear of pain. However, due to the decline in physical exercise, individuals are then at risk for physical deconditioning. By avoiding physical activity, it can in turn cause decreased cardiovascular endurance, loss of strength, loss of endurance, and general decline in health. A general decline in one's health can make them more at risk for their chronic pain to worsen. The cyclic nature of this pattern is referred to as deconditioning syndrome and supports the concept of exercise and fitness reducing chronic pain symptoms (Gupta, et al., 2019). Many varieties of exercise like yoga, Pilates, and other strengthening programs have been added to the literature supporting the efficacy of fitness in pain management (Atalay, Akova, Gur, \& Sekir, 2017; Gupta, et al., 2019; Wu, et. al., 2015).

A study by Wu et al. (2015) states that yoga has been associated with improved posturing, lower stress and anxiety rates, and pain relief. The coupling of mental focus and purposeful positioning bonds the physical and mental components that are impacted by chronic pain. The study also explains the benefits of Pilates and its emphasis on core strengthening, body posturing, coordination, and breathing for relieving chronic pain. The connection between mind and body promotes both physical and mental wellness (Wu, et al., 2015).

Another article written by Atalay, et al. (2017), discusses patients with chronic lower back pain utilizing exercise to relieve pain. One group was given lower back exercises only while the other group used a combined approach of upper and lower back, shoulders, and neck exercises. The group with the combined exercises experienced significantly lower pain and 
disability than those who only performed lower back exercises (Atalay, et al., 2017). The article suggests that combined exercises could be beneficial in lowering chronic back pain.

\section{Conclusion}

When addressing chronic pain with non-pharmaceutical interventions, occupational therapists can bring a variety of options to the table. The different approaches in this section highlight only a few of the most common. There are many other options that could be explored with further research. Utilizing ergonomics, fitness-based programs, adaptive equipment, adaptive strategies, and aquatic therapy do not have to be separate from one another either. The benefit of therapy is that any number of these techniques can be added into a session, or carried out through multiple sessions, to create the best outcome for the patient. They can also be utilized based on the interest of the patient so that therapy can continue to be client centered. These non-pharmaceutical interventions all demonstrate adequate amounts of support as to why they should be utilized in practice. Not only can they be considered useful tools, but they also have support that speaks towards their effectiveness too. There is a clear need for occupational therapists to utilize these tools for chronic pain management intervention.

\section{Chapter Three: Project Description}

\section{Introduction}

The purpose of this capstone was to develop an evidence-based manual for clinicians that outlined non-pharmacological approaches to manage chronic pain symptoms. Occupational therapists are trained to evaluate how pain can influence the way patients interact with their environment and how it can affect their daily occupations (Lagueux, et al., 2018). Chronic pain limits patients' ability to complete everyday tasks, and can affect sleep, appetite, and quality of life (Da Silva Sobrinho, De Almeida, Da Silva Rodrigues, \& Bueno Junior, 2019; Mehta, et al., 
2018). Patients all over the country are living with uncontrolled chronic pain; approximately one in every four Americans currently has chronic pain (Rochman, 2014). Overtime, chronic pain can affect daily occupations and leaves people feeling powerless (Rochman, 2014). Occupational therapists can provide relief to patients experiencing chronic pain (Lagueux, et al., 2018). Occupational therapists can use non-pharmacological approaches to adapt tasks, change task demands, and alleviate pain so a person's daily occupations will once again be possible (Hesselstrand, et al., 2015; Lagueux, et al., 2018).

To help expose the benefits of occupational therapy in chronic pain management, this capstone's focus was on creating an educational evidenced-based manual. The manual created discussed a few specific methods for intervention including body mechanics, aquatic therapy, adaptive equipment, and fitness programs. The manual was also created with patients in mind and included easy to read explanations of the topics at hand. Each topic was supported with recent literature and highlighted the benefits of the intervention and its correlation to OT. According to Lucio et al. (2018) occupational therapy educational programs lack the correlation between what chronic pain is, and an effective method of treating it in everyday practice. This manual aided in addressing the gap between the classroom and the work force for clinicians, advocates for the profession, educates patients, and provided a useful tool to demonstrate the need for the implementation of pain management techniques into everyday OT practice.

\section{Participants}

This capstone project utilized recent literature to create a manual with various nonpharmaceutical OT interventions for chronic pain. A needs assessment was performed through utilization of the staff at the Melbourne Terrace Rehabilitation facility. Various informational sessions were held and were comprised of residents, nursing staff, students, and therapy staff. 
These participants were either personally dealing with chronic pain, treated patients with chronic pain, or simply wanted to expand their knowledge of non-pharmacological OT interventions for chronic pain. It was beneficial to advocate to a wide variety of people who would all utilize the knowledge of occupational therapy's role in chronic pain management a different way. This was useful because it allowed for many different types of people to hear about not only OT, but how either they or their patients could benefit from the utilization of this material.

\section{Setting}

Most of this project was completed at Melbourne Terrace Rehabilitation Facility, a skilled nursing facility (SNF) in Melbourne, Florida. This facility is well known in the area and has a vast array of therapy services available to clients. The residents and staff at the facility made it ideal to gather the participants for the informational sessions. The second place utilized for this project was at the University of Saint Augustine speaking to the students who were part of the Student Occupational Therapy Association (SOTA) during one of their meetings.

\section{Development and Implementation}

Three components were completed during this capstone project. The first one was the conduction of a needs assessment to determine the factors affecting treatment of chronic pain, current intervention utilized, and barriers towards implementation. The second piece was implementation of the informational sessions catered to the audience's connections to OT and framed by the information gathered through the needs assessment. The sessions were provided to onsite to residents and clinicians, as well as offsite students. Session information differed slightly based on the demographic of the audience that was being presented to. The findings from the needs assessment then served as a guide in the creation of the information sessions that were presented to residents, staff members and students during weeks eight through thirteen. The third 
component was the creation of the evidence-based manual utilizing non-pharmacological interventions. This process began during the fifth week on site.

Informational Sessions. In the beginning of week five, appropriate contacts were made in order to schedule the dates for the upcoming informational sessions and necessary arrangements were made for location and time. After the scheduling for the presentations were confirmed, focus was put on completion of the presentations, and to prepare any needed materials. Dates and times were confirmed with the various onsite contacts a couple days prior to the presentation. There were four different informational sessions set to be provided by the end of week 13. Sessions were spaced out appropriately during this time to allow for adequate preparation time for the presentation. The informational sessions did not follow a strict timeline in order to accommodate the sites and to be flexible if the need to reschedule arose. Initially the goal was to complete this by week 13 , however, with some scheduling conflicts, changing of leadership onsite, and other unforeseen circumstances, all informational sessions were completed by week 15 . This still allowed for copious amounts of time to complete all necessary components of the project. After the informational sessions, a Google Form was distributed to receive anonymous feedback regarding the presentation. After the final session was completed in week 15, overall feedback, comments, and concerns were gathered from the Google Form. Any suggestions, comments, or concerns made were utilized when making final edits to the manual.

Building the Manual. During weeks one through four, a literature review was conducted to find appropriate evidence supporting non-pharmaceutical interventions. During the fifth week, the composure of the evidence-based manual began. The manual continued in production until its completion in week 17. Feedback from the site supervisor was ongoing throughout the process via the needs assessment, presentations, and the manual. Feedback on the manual was also 
obtained from the capstone mentor. The manual encompassed all of the capstone components but was heavily focused on advocacy, education, and practical non-pharmaceutical intervention. Most of the manual was composed off site on days that student presence on site was not required. The manual included research on four non-pharmaceutical interventions for chronic pain that included fitness programs, adaptive equipment, aquatic therapy, and the use of proper body mechanics. Other components of the manual included information on chronic pain definitions, statistics, and explanations of opioid usage. The manual also has an interactive component for users to apply the knowledge they have read that includes a crossword puzzle, word search, and word matching activities.

Dissemination of Project. Once the informational sessions and manual were completed, a copy of the manual was given to the student's site supervisors at Melbourne Terrace Rehab. The manual is to serve as a useful tool for the therapy staff for both patients and rehabilitation staff at the facility. The goal of providing this manual to the staff was to provide a concrete resource to utilize with their residents who could benefit from reduction of chronic pain.

\section{Evaluation and Revision}

In order to revise the effectiveness of the informational sessions provided, the student used a survey via Google Forms to obtain necessary feedback. The use of feedback was helpful for future sessions because it gave the student the opportunity to add additional information the audience would have liked to know. The feedback was also utilized when forming the manual to be sure to address areas of interest. The survey was anonymous to participate in but had a question to identify which of the informational sessions was attended. This was the best way to grasp which demographic was answering and how they responded to the information provided. 
The informational sessions were all provided to the correct audiences in a timely manner. All but the nursing staff presentation were provided in person. Due to unforeseen circumstances the format of the nursing staff presentation had to be transitioned to a virtual one. A recorded PowerPoint presentation was sent to the staff development coordinator as the alternative solution. The link to the Google Form was also sent along with the presentation.

In addition to the feedback from the Google Form, feedback was also provided from the site supervisor and capstone mentor on an as needed but ongoing basis. The element of feedback was important to this project because a key element was education. The informational sessions and the manual were created with educating its users in mind. The goal being to create a product that is easy to understand and practical to utilize. Feedback from the participants served as a helpful tool during this process in order to create the most efficient tool. Participants said not only was the information in the sessions helpful for future practice, it was something that could be taken with them in current practice. Other feedback from the site supervisor stated that this project had significant weight in current practice. The site supervisor said this is because addressing pain is becoming something clinicians are realizing is extremely important for quality patient care. Other therapy staff said that it was useful and simply put so that it did not seem so daunting to address in an everyday manner. The only real adverse comments were not negative at all, but merely wishes of those who had hoped for touching on more topics involving pain. Most of those requests did not fit into the scope of this capstone. Overall, the presentations were received well, and feedback was positive.

\section{Alignment with Objectives}

The objectives of this capstone were completed through the evaluation, creation, and dissemination of an evidence-based resource manual on chronic pain. During the evaluation 
stages a needs assessment was performed to find the most common types of chronic pain at the SNF. Therapists, residents, and other employees were observed to best represent the needs of the population. In addition, through the utilization of the needs assessment results, four informational sessions were developed to educate the residents, students, nursing staff, and therapy staff on the benefits of OT in chronic pain management. Session feedback was utilized to promote a well-rounded evidence-based manual that would represent various interventions and could be understood by both patients and clinicians alike.

During the creation phase the manual was put together remotely in the time the student was not on site. The manual included recent literature supporting the use of non-pharmacological interventions such as body mechanics, aquatic therapy, fitness programs, and the use of adaptive techniques. The manual also included interactive materials, statistics, and information about chronic pain. This manual has been revised with feedback from the sessions, as well as through feedback from the capstone mentor.

During the final dissemination phase, the final product was given to the facility to be utilized by occupational therapists when addressing chronic pain, but also to serve as a tool for educating patients on the topic. The goal of the manual is to further advocate for the occupational therapy clinician's role in chronic pain. In addition, the manual sought to bring more comfortability to therapists who utilize these tools in practice while also serving as a resource for patients to learn more about OT and their role in addressing chronic pain symptoms.

\section{Conceptual Framework}

The logic model created a frame to lay out the capstone project in a well thought out manner. According to Sousa, Shoemaker, Nascimento, Costa, and Ramalho de Oliveira (2018) the logic model uses analyzing factors called inputs to control the activities utilized in the 
creating of the product, and it addresses what the outcome would be. Inputs are the resources that were utilized for the project at hand. Inputs for this capstone are the utilization of the SNF and its faculty, technology, and recent literature. The activities are steps that were performed during the project. These included the conduction of the needs assessment, formation of the manual, and creation of the informational sessions. The product, or output, is what was provided to the facility. In this case, the manual on non-pharmaceutical interventions for chronic pain was the product. Outcomes are the changes or benefits that are foreseen with this project (Sousa, et al., 2018). For this capstone the outcomes were to advocate for occupational therapy's role in chronic pain, to educate various populations about how OT influences chronic pain management, and to provide a practical tool for applying non-pharmacological interventions in everyday OT practice. For the short-term perspective these outcomes appeared to be more immediate to the direct impact of the facility. However, the hope was to be able to generalize the manual to a community dwelling population to have greater impact in the chronic pain epidemic and on the field of occupational therapy. This project aimed to influence the way various groups of people view OTs role in chronic pain.

\section{Timeline}

The first five weeks on site, time was spent performing a needs assessment on the prevalence, incidence, and etiology of chronic pain. Information was gathered by shadowing therapists, patients, and other clinicians to establish the most common chronic pain symptoms and diagnoses present at the SNF. During weeks five through fifteen the manual was created. Between weeks eight and thirteen, four informational sessions were provided to the patients, students, therapy staff, and nursing staff members. Information was spread via the use of the student's site supervisor. The site supervisor connected the student to a staff development 
coordinator and the activities director to plan the informational sessions with them for nursing and residents. The student informational session was planned through the professional development position on the SOTA board at the university. All of these connections made it possible to accurately target the correct populations for the presentations. Each of the points of contact were able to advertise the informational sessions to assure adequate attendance to each session by planning them during already existing meetings or in-service dates. This helped tremendously regarding the need for advertisement.

In the fourteenth week, the manual was in it its final stages. Weeks thirteen through fourteen were used for additional feedback, revisions, clarifications, and final changes. This allotted time gap allowed for a more polished product. It also ensured that even with any unforeseen setbacks, the manual was still completed within its proper time constraints. At the end of week fourteen, all work on the evidence-based resource manual was completed. The information gathered for the manual was ready to be given to the staff at Melbourne Terrace Rehabilitation. During week fifteen the manual was disseminated to the site supervisor, and a copy of the final product was left with the company as a future resource.

\section{Outcome and Future Implications}

The result of this capstone project is an evidence-based manual created to leave with the clinicians at Melbourne Terrace Rehab. The hope is that this resource will be generalizable to the community population and be able to be dispersed elsewhere to continue as a resource for patients, students, and therapists. As a direct result of this capstone, the goal is to bring awareness to shocking prevalence of chronic pain and the underwhelming use of nonpharmaceutical intervention. This capstone has clearly acknowledged the need for occupational therapy in the non-pharmaceutical intervention for chronic pain. Moving forward it will serve as 
a tool to increase the awareness of clinicians of occupational therapy and more to the importance of this matter and it's necessity to be addressed.

With 70 million people diagnosed with chronic pain, and many of them experiencing little to no relief while using opioids it was obvious that there needed to be more evidence added to the literature providing a solution to the problem (CDC, 2017; Hadlandsmyth, et al., 2018). This capstone project chose to focus on occupational therapy's ability to utilize nonpharmacological approaches. This project aimed to shed light on an occupational therapist's ability to reduce their patient's need for pharmaceutical interventions. This project also displays the duty that occupational therapists have to their patients who are experiencing chronic pain. Occupational therapists, using fitness-based exercise, adapted equipment, adapted strategies, aquatic therapy, and ergonomics can lower the need of opioid drugs for people managing chronic pain all while increasing their independence and quality of life. This project adds to the literature supporting occupational therapy's role in chronic pain management by using recent and relevant literature. The project also serves to educate patients and clinicians of all kinds, and advocates for occupational therapy's ability to better serve the chronic pain population.

The future implications of this manual may involve publication in OT Practice Magazine or on the American Occupational Therapy Association's website. Other possibilities include presenting to other students and clinicians at the University of St. Augustine or local state conferences. Another use for the manual is to be a tool to inspire and educate clinicians and patients alike for OTs role in chronic pain management. The main goal is to provide a resource that is functional, educational, and practical to apply in everyday OT life. This resource should be able to be utilized even after the capstone ends and should be able to be used across a variety of settings with a variety of people. 


\section{Chapter Four: Results and Analysis}

\section{Product/Project}

Informational Sessions. The informational sessions provided were given to four different groups: nursing staff, students, therapy staff, and patients. These informational sessions served as education and advocacy for occupational therapy's role in chronic pain and how it applied to that specific audience. Originally the informational sessions were going to be given only to older adults at the facility. However, it became clear after the conduction of the needs assessment that therapy staff, nursing staff, and students would also benefit from these discussions because they all deal with chronic pain every day either personally or professionally. With the approval of the site supervisor and capstone mentor, the objective was shifted from only including older adults to including a more diverse population of individuals.

The purpose of broadening the scope was to promote inter-disciplinary education on occupational therapy's role in chronic pain. For example, if the nursing staff members are more aware of the supportive role occupational therapists play for their patients who have chronic pain, they may be more likely to recommend the patient to therapy instead of solely providing pain medicine. Using a non-pharmaceutical approach could assist the patient in becoming more independent, thus not only increasing their quality of life, but also reducing the caregiver burden on the nursing staff. Likewise, if therapists are given research-based intervention ideas as well as practical examples of application, treating chronic pain may not seem as daunting. If there can be education and advocacy for students, it can be assumed that they can then take this knowledge into practice when they go. Education and advocacy became a lot more prominent as the needs assessment results were processed. It was clear that there needed to be practical, applicable, research-based information presented to these populations. By being able to provide the 
informational sessions to a variety of audiences the reach of the project was expanded for the better.

Three informational sessions were completed in person on site. The students, therapy staff, and resident presentations were all completed in person at the facility or at the university. The Google Form was provided after each presentation to receive feedback. Due to unforeseen circumstances, the final informational session scheduled for nursing staff required being completed virtually via recorded PowerPoint presentation. The minor change did not affect the quality of the presentation. The presentation was sent to the staff development coordinator to be presented to the clinicians as the next best option. The Google Form was still provided to the staff to complete after watching the video presentation.

Resource manual. Another component of this capstone was the production of the evidence-based manual. This tool was formed to be a resource for not only patients but clinicians as well. The manual was created with patient friendly language to be easy to understand as well as with research-based components to serve as a tool for occupational therapists in practice. The resource is broken into easy-to-read chapters and provides a helpful look into what chronic pain is, how it is treated, what OT is, and non-pharmaceutical approaches. This manual was developed to be informative to both patients and clinicians alike. The manual concepts did not shift much over time. The main concept was to form an evidence-based tool that was inclusive of any population.

\section{Measurement of Effectiveness}

Being able to measure the effectiveness of the project is important to the success and carry over of the educational components of the capstone. A brief survey was provided after the presentation via Google Forms. The survey was anonymous and took about 5 minutes to fill out. 
Participants were asked if the role of OT was clearly explained in relation to chronic pain management, if there was any further information they would have wanted to know, and for their feedback, comments, and concerns. The consensus from the informational session feedback determined that the information provided was helpful, concise, and delivered effectively by the student. It was noted that they would have liked more information on how to identify patients that may be relying too heavily on pharmaceuticals. Participants also wanted information on the medication management side of therapy for patients who are already taking other medications. While this information can be helpful when delivering occupational therapy services, it is slightly outside of the context of this project as the focus is on non-pharmaceutical intervention.

The focus of the initial project was geared more towards residents to educate them on the role of OT in chronic pain. However, the needs assessment made it clear that while it was beneficial to share this with patients, there was a huge need to advocate for this role with clinicians as well. In fact, it was evident that utilizing non-pharmaceutical pain management was highly overlooked during day to day practice. The needs assessment unveiled the necessity of this capstone to bring a practical awareness of OT's role in chronic pain.

Despite the facility being fully equipped with antigravity treadmills, two therapy pools, and other resources to address chronic pain, the common practice mostly focused on the acute pain at hand. It was noted in the needs assessment that the clinicians were doing great work solving problems to the patients' current acute pain struggles, but when asked about how they manage chronic pain, the answers from occupational therapists and physical therapists onsite were all similar. The most common reason noted for not implementing treatments, like the antigravity treadmill or the aquatic therapy pools, was the burden of the equipment. Therapists often said that because it took so long to get the patient in and out of the equipment, using such 
an intervention can take up most of their sessions. Therapists said that they wanted to make sure the patient was receiving the most effective services possible by using multiple techniques rather than just one for the entire session.

The second and third reasons, found to be of equal importance, were the patient having the physical ability to use the specific equipment and the contraindications the patient might have as to why they are not a suitable candidate in the first place. For example, to use aquatic therapy services in therapy the patient is not allowed to have open wounds and they must be fully continent. According to the therapists on site, these are the most common reasons why patients were not a match for the intervention. The findings of the needs assessment provided evidence that supported the necessity of this capstone project for advocating OTs role in chronic pain. It became apparent that regardless of the information therapists, students, nursing staff, and patients know about occupational therapy, they may not be aware of all the small components that can be covered or how to implement them in a practical manner.

After the informational sessions occurred, the feedback from the Google Form was utilized to make edits and various modifications to the manual. This was to ensure that the manual would encompass everything that people would have wanted to know about chronic pain, opioids, interventions, and occupational therapy. The overall feedback was that the informational sessions were provided in an easy to understand and concise manner. Participants felt as though the information would benefit them in the future.

\section{Results/Discussion}

Education. After presenting the informational sessions and completing the manual, the main outcomes of the project were education, product development, and advocacy for the profession of occupational therapy, specifically for non-pharmaceutical intervention. This project 
has created a space to inform current and future clinicians about how occupational therapy can be an extremely beneficial asset to treating chronic pain. While the research being collected into one resource is a stand-alone piece that can serve as a useful tool, being able to bring mini informational sessions to various populations allowed for the research and awareness of the topic to be further spread. Having a combination of how the information is provided is effective and helpful.

Advocacy. This project was heavily focused on advocating the important role occupational therapy can have on management of chronic pain with the use of nonpharmaceutical interventions. It allowed for a wide range of people to understand a perspective that would benefit them in their future practice as well. Based on the feedback of this project, it was effective in representing a practical way to implement more occupational therapy in this area.

Resource Manual. The resource manual ties these two components of advocacy and education together into a useful and practical tool for not only clinicians but also patients. It allows for the information of research and patient friendly language to merge and create a tool that is informative while also allowing for the clinician to utilize it in practice. It was created to be practical, useful, and handy to have in a clinic or in a waiting room of a therapy office.

\section{Implications}

The implications of this capstone added to the occupational therapy literature, advocated for occupational therapist's role in chronic pain management, and presented evidence-based support for non-pharmaceutical approaches to chronic pain management. This project was significant in making this niche of a project area more well known. This project created a resource that is easy to use by patients and clinicians alike. Increasing the practice of non- 
pharmaceutical intervention for chronic pain management has the potential to lower the need for patients to use pharmaceuticals, increase their independence, and potentially reduce their pain. This project highlighted the research and demonstrated support for occupational therapy's role in chronic pain management.

\section{Limitations and Delimitations}

One limitation addressed during this capstone project was the availability of people to attend the informational sessions which could have resulted in reduced attendance due to busy schedules of staff, students, and residents. Another limitation was that since this project was completed in a facility, it may not have been generalizable to the community-dwelling population. In addition, a third limitation was there was no control over which diagnoses, or comorbidities were present in the SNF population.

A delimitation of this project was the choice to use an older adult population, for this project older adult will refer to those 65 years and older. This is due to age being a big factor influencing chronic pain. Another delimitation was choosing to implement this project at a SNF as opposed to another type of clinic or in a hospital setting.

\section{Chapter Five: Summary, Conclusions, and Discussion}

\section{Summary}

This capstone project aimed to add to the literature demonstrating why occupational therapy should be an asset in treating chronic pain and what types of evidence-based interventions support this notion. This capstone has clearly established that occupational therapists are capable of such treatment and intervention (Amris, et al., 2019). It has also displayed a multitude of research supporting the types of interventions and their benefits for people living with chronic pain. Throughout this capstone it has been made plain that 
occupational therapists can and should be a key part in the non-pharmaceutical intervention approach to chronic pain (Lagueux, et al., 2018). Occupational therapists combine their ability to adapt, create, and grade tasks by using adaptive equipment, adaptive strategies, aquatic therapy, fitness-based exercise, and ergonomics so patient's pain can be lowered while also increasing their independence and quality of life.

This project exposed the strengths and necessity of occupational therapy in chronic pain management. The capstone chose to focus on advocacy for the profession and education on OT's role in chronic pain. These two components were used to build a manual that allowed for the information to have a lasting effect even after the project was completed. The manual aided in addressing the gap between classroom and everyday treatment sessions for by acknowledging what types of interventions can successfully be utilized in therapy (Lucio, et al., 2018). This project also provided personal information sessions to various audiences to further the advocacy of occupational therapy's role in chronic pain. The informational sessions allowed for a wider spread of education throughout the facility while the manual will provide a lasting tool for advocacy and education in the future.

\section{Strengths and Challenges}

This capstone project presents several strengths. This project successfully utilized information from a student-conducted needs assessment to reinforce the necessity of occupational therapy's role on chronic pain. Four informational sessions were presented successfully to nursing staff, therapy staff, current students, and residents. The evidence-based manual was completed and serves as a tool to connect education, information, advocacy, and entertainment in one. The manual was created in a PDF format that will be easily accessible to send to companies and/or print copies from if needed in the future. The project not only added to 
the literature but will continue to serve as a tool for advocacy and education after the project is completed.

Finishing the capstone project held challenges as well. The main one being the availability of participants for the informational sessions. Finding time that could be properly allotted without interfering with work schedules of the staff was difficult but not unattainable. The site and the student were both compromising and flexible on this matter. The other challenge was creating a manual that would be patient friendly as well as useful to the therapists. Finding the balance of appropriate language while remaining educational posed difficult at times. However, despite these challenges, the project was still a success and added to the literature for occupational therapy's role in chronic pain management.

\section{Future Considerations}

In the interest of being a relevant and useful tool, this project was created with the ability to be translated to any population setting that is dealing with chronic pain. The project itself may have been completed in a skilled nursing setting, but chronic pain affects many people of a wide range of ages and the project would be relevant for most. Moving forward, the hope is that the project can be published either virtually or in print form to be able to continue to spread the word about occupational therapy's role in the chronic pain realm. The student's hope is to be able to publish the manual and present the information at the FOTA conference in the future.

\section{Conclusion}

When this project was in the beginning stages, its intention was to bring awareness of occupational therapy's role in chronic pain to light for the older adult population so that it could pose an alternative to pharmaceutical approaches. These proposed interventions were then supported by evidence-based literature and presented to a variety of audiences. Initially the 
project was to educate only older adults at the skilled nursing facility. However, with the addition of the other audiences, it has created a more inclusive project while also expanding the advocacy and education component's reach. The capstone now incorporates four subsections of people receiving information and education on how occupational therapy can affect chronic pain, as well as how it can help them. By making more people aware of all that occupational therapy can offer in relation to pain management, an opportunity is provided to help more patients who are living with chronic pain.

The manual has become a useful tool for patients and clinicians alike and can be easily received in a wide variety of contexts. Even with the changes, the student was able to maintain the objectives of this capstone while also being able to expand it to impact a greater population. The advocacy factor of this capstone was also more expansive than initially considered. It became evident that advocating for occupational therapy's role in non-pharmaceutical interventions for chronic pain was something that needed to be done. By educating various audiences to understand what occupational therapy can do, it highlights a more holistic and medication free alternative. While this cannot guarantee an elimination of medication, since that is not an occupational therapist's scope, it can pose an alternative approach to giving a patient more independence and a better quality of life. Having a greater use of occupational therapy in this way directly reflects purpose of the capstone. This capstone is successfully adding to the literature while increasing awareness so that ultimately pain can be reduced, independence can be restored, and quality of life can be returned to patients. In turn, by implementing a more nonpharmaceutical approach, hopefully it will decrease the need for excessive pharmaceutical interventions in the future. 
Taking what was created in this capstone and looking forward, there is a great potential to take the research and the manual created to conferences or to publish it in some capacity, be that virtual media or print. It is something that is a prevalent issue throughout many settings and needs to continue to be discussed. The manual adds to the literature supporting the necessity of using occupational therapy in treating chronic pain. It should be used not only in the occupational therapy world but to advocate in the rest of the medical world too. The hope is that the manual will be continuously utilized tool that helps further the reach of this project into the occupational therapy community, the medical community, and the chronic pain community in a way that will continue advocating and educating long after the project has been completed. Through doing so, this project will serve as an example to occupational therapists and their patient as to why OT is so valuable in chronic pain management. 


\section{References}

Almutairi, A. R., Mollon, L., Lee, J., \& Slack, M. (2019). A comparison of the pharmacologic and nonpharmacologic strategies used to manage chronic pain: Opioid users versus nonusers. Journal of the American Pharmacists Association, 59(5), 691-697. https://doi.org/10.1016/j.japh.2019.06.009

Amris, K., Bülow, C. von, Christensen, R., Bandak, E., Rasmussen, M. U., Danneskiold-Samsøe, B., \& Waehrens, E. E. (2019). The benefit of adding a physiotherapy or occupational therapy intervention program to a standardized group-based interdisciplinary rehabilitation program for patients with chronic widespread pain: A randomized activecontrolled non-blinded trial. Clinical Rehabilitation, 33(8), 1367-1381. https://doi.org/10.1177/0269215519843986

Atalay, E., Akova, B., Gür, H., \& Sekir, U. (2017). Effect of upper-extremity strengthening exercises on the lumbar strength, disability and pain of patients with chronic low back pain: A randomized controlled study. Journal of Sports Science \& Medicine, 16(4), 595603. Retrieved from http://search.ebscohost.com/login.aspx?direct=true \&db=s3h\&AN=126631601\&site=edslive

Birke, H., Ekholm, O., Højsted, J., Sjøgren, P., \& Kurita, G. P. (2019). Chronic pain, opioid therapy, sexual desire, and satisfaction in sexual life: A population-based survey. Pain Medicine, 20(6), 1132-1140. Retrieved from http://search.ebscohost.com/login.aspx?direct=true \&db=s3h\&AN=136864181\&site=edslive 
Centers for Disease Control and Prevention. (2019). Opioid overdose. Retrieved from https://www.cdc.gov/drugoverdose/index.html

Cheng, M. (2017). New management strategies for patients with chronic pain. Journal of Managed Care Medicine, 20(3), 20-25.

Da Silva Sobrinho, A. C., De Almeida, M. L., Da Silva Rodrigues, G., \& Bueno Júnior, C. R. (2019). Association of chronic pain with strength, levels of stress, sleep and quality of life in women over 50 years. Fisioterapia e Pesquisa, 26(2), 193-200. https://doi.org/10.1590/1809-2950/18033226022019

Dahl-Popolizio, S., Manson, L., Muir, S., \& Rogers, O. (2016). Enhancing the value of integrated primary care: The role of occupational therapy. Families, Systems \& Health, 34(3), 270. Retrieved from http://search.ebscohost.com/login.aspx?direct=true \&db=edsgsr\&AN=edsgcl.465558430 $\&$ site=eds-live

Dahlhamer, J., Lucas, J., Zelaya, C., Nahin, R., Mackey, S., DeBar, L., ... Helmick, C. (2018). Prevalence of chronic pain and high-impact chronic pain among adults. Morbidity and Mortality Weekly Report. 67(36), 1001-1006. Retrieved from: http://dx.doi.org/10.15585/mmwr.mm6736a2

Domenichiello, A. F., \& Ramsden, C. E. (2019). The silent epidemic of chronic pain in older adults. Progress in Neuropsychopharmacology \& Biological Psychiatry, 93, 284-290. https://doi.org/10.1016/j.pnpbp.2019.04.006

Gupta, P., Mohanty, P. P., \& Pattnaik, M. (2019). The effectiveness of aerobic exercise program for improving functional performance and quality of life in chronic low back pain. Indian 
Journal of Physiotherapy \& Occupational Therapy, 13(2), 155-160. https://doi.org/10.5958/0973-5674.2019.00064.9

Hadlandsmyth, K., Stewart, K. R., Paez, M. B., Steffen, M., Meth, M., Reisinger, H. S., \& Mosher, H. J. (2019). Patient perspectives on opioids: Views of inpatient veterans with chronic pain. Pain Medicine, 20(6), 1141-1147. Retrieved from http://search.ebscohost.com/login.aspx?direct=true \&db=s3h\&AN=136864197\&site=edslive

Hasanpour-Dehkordi, A., Dehghani, A., \& Solati, K. (2017). A comparison of the effects of Pilates and McKenzie training on pain and general health in men with chronic low back pain: A randomized trial. Indian Journal of Palliative Care, 23(1), 36-40. https://doi.org/10.4103/0973-1075.197945

Hesselstrand, M., Samuelsson, K., \& Liedberg, G. (2015). Occupational therapy interventions in chronic pain - A systematic review. Occupational Therapy International, 22(4), 183. https://doi.org/10.1002/oti.1396

Hill, W. (2016). The role of occupational therapy in pain management. Anaesthesia \& Intensive Care Medicine, 17(9), 451-453. https://doi.org/10.1016/j.mpaic.2016.06.008

Hill, W., \& Macartney, M. (2019). The role of occupational therapy in enabling people with chronic pain to return to work or education. Anaesthesia \& Intensive Care Medicine. 20(8), https://doi.org/10.1016/j.mpaic.2019.05.007

Keane, L. G. (2017). Comparing AquaStretch with supervised land-based stretching for chronic lower back pain. Journal of Bodywork \& Movement Therapies, 21(2), 297-305. Retrieved from 
http://search.ebscohost.com/login.aspx?direct=true\&db=s3h\&AN=123132006\&site=edslive

Lagueux, É., Dépelteau, A., \& Masse, J. (2018). Occupational therapy’s unique contribution to chronic pain management: A scoping review. Pain Research \& Management, 1-19. https://doi.org/10.1155/2018/5378451

Lauche, R., Stumpe, C., Fehr, J., Cramer, H., Cheng, Y. W., Wayne, P. M., Rampp, T., Langhorst, J., \& Dobos, G. (2016). The effects of tai chi and neck exercises in the treatment of chronic nonspecific neck pain: A randomized controlled trial. Journal of Pain, 17(9), 1013-1027. https://doi.org/10.1016/j.jpain.2016.06.004

Lucio, A., Laney, J., Lake, S., Kornblau, B., \& Mbiza, S. (2018). Occupational therapy interventions for the treatment of pain. American Journal of Occupational Therapy, 72, 1. Retrieved from http://search.ebscohost.com/login.aspx?direct=true \&db=edo\&AN=134729848\&site=edslive

McIlroy, S., Sayliss, L., Browning, P., \& Bearne, L. M. (2017). Aquatic therapy for people with persistent knee pain: A feasibility study. Musculoskeletal Care, 15(4), 350-355. https://doi.org/10.1002/msc.1179

Mehta, P., Kaur, M., Smith, C. M., Mani, R., \& Baxter, G. D. (2018). Aging well with chronic musculoskeletal pain: Protocol for a systematic review of non-pharmacological interventions aimed at reducing pain in an ageing population. Physical Therapy Reviews, 23(6), 330-337. https://doi.org/10.1080/10833196.2018.1540167 
Murray, M., Stone, A., Pearson, V., \& Treisman, G. (2019). Clinical solutions to chronic pain and the opiate epidemic. Preventive Medicine: An International Journal Devoted to Practice and Theory, 118(2019), 171-175. https://doi.org/10.1016/j.ypmed.2018.10.004

Radomski, M.V., Trombly Latham, C.A. (2014). Occupational therapy for physical dysfunction, Seventh Edition, Philadelphia, PA. Lippincott. ISBN; 978-14511-2746-1

Rochman, D. (2014). Occupational therapy and pain rehabilitation. American Occupational Therapy Association (AJOT). Retrieved from https://www.aota.org/ /media/Corporate/Files/AboutOT/Professionals/WhatIsOT/HW/Fa cts/Pain\%20Rehabilitation\%20fact\%20sheet.pdf

Russell, N., Daniels, B., Smoot, B., \& Allen, D. D. (2019). Effects of yoga on quality of life and pain in women with chronic pelvic pain: Systematic review and meta-analysis. Journal of Women's Health Physical Therapy, 43(3), 144.

Simon, A., \& Collins, C. (2017). Lifestyle Redesign® for chronic pain management: a retrospective clinical efficacy study. American Journal of Occupational Therapy, 71(4), 1-7. https://doi.org/10.5014/ajot.2017.025502

Simsek, N., Evli, M., \& Uzdil, N. (2019). Learned resourcefulness, anxiety and depression levels in patients with chronic pain. International Journal of Caring Sciences, 12(1), 240-247. Retrieved from http://search.ebscohost.com/login.aspx?direct=true\&db=ccm\&AN=136698189\&site=eds -live

Sousa, S., Shoemaker, S., Nascimento, M., Costa, M., \& Ramalho de Oliveira, D. (2018). Development and validation of a logic model for comprehensive medication management 
services. International Journal of Pharmacy Practice, 26(3), 250-257. https://doi.org/10.1111/ijpp.12392

Stensland, M. L., \& Sanders, S. (2018). Not so golden after all: The complexities of chronic low back pain in older adulthood. Gerontologist, 58(5), 923-931. https://doi.org/10.1093/geront/gnx154

Vendrusculo-Fangel, L., Fangel, R., dos Santos Leles, T., de Melo Moura, L., \& de Cássia Marqueti, R. (2019). Changes in occupational roles in women with chronic pain and rheumatoid arthritis compared to healthy women. Brazilian Journal of Occupational Therapy, 27(1), 135-148. https://doi.org/10.4322/2526-8910.ctoAO1798

Weissenfels, A., Wirtz, N., Dörmann, U., Kleinöder, H., Donath, L., Kohl, M., Fröhlich, M., von Stengel, S., \& Kemmler, W. (2019). Comparison of whole-body electromyostimulation versus recognized back-strengthening exercise training on chronic nonspecific low back pain: A randomized controlled study. BioMed Research International, 1-9. https://doi.org/10.1155/2019/5745409

Wu, P. I.-K., Meleger, A., Witkower, A., Mondale, T., \& Borg-Stein, J. (2015). Nonpharmacologic options for treating acute and chronic pain. $P M \& R, 7(11)$, S278S294. https://doi.org/10.1016/j.pmrj.2015.09.008

Yaban, Z. S. (2019). Usage of non-pharmacologic methods on postoperative pain management by nurses: Sample of turkey. International Journal of Caring Sciences, 12(1), 529-541. Retrieved from http://search.ebscohost.com/login.aspx?direct=true\&db=ccm\&AN=136698222\&site=eds -live 
Zamuner, A. R., Andrade, C. P., Arca, E. A., \& Avila, M. A. (2019). Impact of water therapy on pain management in patients with fibromyalgia: Current perspectives. Journal of Pain Research, 1971. https://doi.org/10.2147/JPR.S161494 